\section{Ein Fall von Tracheostenose und plötz- lichem Tode durch Thymusschwellung.}

\author{
Von Dr. Hermann Cohn in Berlin.
}

Ueber die Frage, ob durch Thymusschwellung Atmungsstörung, Tracheostenose erzeugt werden kann und in welchem Zusammenhange plötzliche Todesfälle kleiner Kinder mit Thymusschwellung stehen, sind in der Literatur zahlreiche Arbeiten vorhanden. Im allgemeinen bezogen sich diese Arbeiten auf Fälle, in denen Kinder plötzlich und ohne vorausgegangene Krankheitserscheinungen starben und bei der Sektion Thymusschwellung gefunden wurde, viel seltener wurde über Fälle berichtet, wo zu Lebzeiten Tracheostenose und Zirkulationsstörungen beobachtet wurden, und danach durch Operation oder Sektion Thymusschwellung gefunden wurde. Sichere Fälle dieser Art sind nur die von Sigel, Biedert und Hans Cohn berichteten. Da klinische Beobachtungen über Druckwirkung der Thymus auf die Nachbarorgane zur Beurteilung der eingangs genannten Fragen von großer Bedeutung und wichtiger noch als Sektionsergebnisse sind, so teile ich einen von mir beobachteten Fall mit.

Am 18. Mai 1906 wurde ich zu dem acht Monate alten Kinde L. gerufen. Nach der Erzählung der Mutter war das Kind schon seit vier Wochen etwas schweratmig, diese Schweratmigkeit hätte sich seit 14 Tagen verstärkt, gleichzeitig hätte das Kind unregelmäßig gefiebert, seit vier Tagen sei die Atmung schnarchend. In den letzten zwei Tagen habe das Kind öfter krampfartige Anfälle gehabt. Dieser letzteren wegen wurde ich gerufen; bisher hatte ärztliche Beobachtung nicht stattgefunden. Das Kind war gut genährt, fett, blaß, etwas pastös. Sogleich auffällig war, daß das Kind stenotisch atmete, Einatmung und Ausatmung gingen mit schnarchendem Geräusch einher, bei der Einatmung waren deutliche inspiratorische Einziehungen der seitlichen, unteren Thoraxflächen vorhanden, rhythmisch traten bei der Atmung, besonders im Exspirium, über dem Jugulum und besonders auf der linken Seite des Halses polsterartige Schwellungen hervor. Die Lippen waren rot, nicht cyanotisch.

Der Anblick erweckte natürlich sofort den Verdacht einer diphtherischen Stenose oder eines Retropharyngealabscesses. Indessen von beiden war nichts zu finden, Hals und Nase waren frei, im Rachen keine Schwellungen palpabel, akute Cervicaldrüsenschwellungen bestanden nicht, der Kopf war frei beweglich, der Husten nicht laryngeal, nicht bellend. Auf den Lungen Erscheinungen einer erheblichen, diffusen Bronchitis. Mangels einer Erklärung für die Stenose und beeinflußt durch eine Gedunsenheit der unteren vorderen Halspartien und die rhythmischen Anschwellungen über dem Jugulum palpierte ich die unteren Halspartien näher. In der Fossa jugularis hatte man das Gefühl, als ob sich vom Mediastinum her bei der Atmung eine Resistenz nach oben verschiebe, bei der Perkussion fand sich über dem oberen Teil des Sternum eine deutliche und starke Dämpfung, die das Sternum nach beiden Seiten etwas überschritt und bis zur Höhe der dritten Rippe reichte. Temperatur war $38,8^{\circ}$, Puls voll und kräftig, 140 .

Nach alledem diagnostizierte ich Atmungsstörung durch Thymusschwellung und ordinierte Jodtinkturpinselung auf den oberen Teil des Sternums und hydropathische Umschläge, kreuzweis von den Schultern nach dem Sternum mit wollener Bedeckung, innerlich Ipecacuanhainfus.

Beim Besuch am nächsten Tage war der Befund unverändert, nur erzählte die Mutter jetzt sicher, daß das Kind alle zwei bis drei Stunden einen Anfall bekäme, in dem es blau wurde und fast sterbe. Während ich die stenotisch-schnarchende Atmung des Kindes betrachtete, trat unter meinen Augen der Anfall ein. Das Kind begann plötzlich ängstlich zu wimmern, mit den Händen unruhig um sich zu schlagen, den Kopf zu drehen, der vorher volle und kräftige Puls wurde sehr klein, fadenförmig und sehr beschleunigt, über 200 in der Minute, die Lippen wurden bläulich und schließlich tief cyanotisch. Auf diesem Höhestadium der Asphyxie verharrte das Kind wenige Sekunden, dann wurde der Puls wieder kräftiger, die Lippen wieder rot, die stieren Augen wieder lebhaft, und nach insgesamt zwei Minuten war alles vorüber; das Kind atmete zwar stenotisch wie zuvor, war aber sonst munter. Eine Steigerung der Stenose in diesem Anfall war nicht vorhanden, es war ganz augenscheinlich ein Anfall von Herzstörung. Die Temperatur war jetzt $40^{\circ}$ in ano. Die Behandlung wurde in gleicher Weise fortgesetzt. In der nächsten Nacht wurde das Kind dann von den Eltern tot in seinem Bett gefunden. Die Anfalle hatten sich oft wiederholt, und in einem der Anfälle war das Kind geblieben. Einer Aufforderung, das Kind zu einer eventuellen Operation in ein Krankenhaus zu bringen, hatten die Eltern nicht nachkommen wollen.

Auf mein Ersuchen wurde mir erlaubt, in kleinem Maßstabe und ohne daß der Leiche ein Teil entnommen würde, eine Sektion zu. machen.

Schnitt vom Kehlkopf bis unter den Nabel, Durchtrennen der Rippenknorpel, Abheben der vorderen Brustwand. Bei dieser Abhebung entleerte sich ein Strom Eiter, etwa $20 \mathrm{~g}$, wie sich herausstellte, ans einem angeschnittenen AbsceB der Thymus. Die Thymus war in einen kleinapfelgroßen Eitersack verwandelt, nur der obere Teil hatte seine drüsige, lappige Beschaffenheit noch, am unteren Teil des Eitersackes war der Herzbeutel adhärent. Eiterungs- oder Schwellungsprozesse an anderen Organen des Mediastinum wurden nicht bemerkt. Eine nähere Untersuchung der Trachea, der großen Gefäße, des Herzens wurde leider nicht vorgenommen; in den Pleuren nichts Abnormes, die Lunge zeigte keine Infiltrationen.

Wenn ich der Mitteilung des Falles eine kurze Epikrise anschließe, so kann ja nicht zweifelhaft sein, daß die gefundene Thymusvereiterung die Ursache der Symptome, die das lebende Kind bot, war. Der Fall bietet also ebenso wie die von Sigel und Biedert beschriebenen einen Beweis dafür, daB Tracheo* stenose durch Thymusschwellung möglich ist. Viele von den Autoren, die sich mit Thymusschwellung beschäftigten, leugneten die Möglichkeit einer Tracheostenose, die einen aus anatomischen Gründen, weil vor der Trachea sozusagen als Schutzwall gegen die hypertrophische Thymus die Wurzeln der großen Gefäße gelegen seien; die anderen mit Rücksicht auf Experimente von Scheele, nach denen erst bei ziemlich starker Belastung der Trachea Atmungsstörungen beim Kaninchen eintraten. Solche Experimente können selbstverständlich nie die natürlichen Verhältnisse nachahmen und beweisen deswegen wenig gegenüber klinischen Erfahrungen, wie sie in den Fällen von Sigel, Biedert und in meinem Falle vorlagen. Immerhin lehren sowohl die seltene Beobachtung von Tracheostenosen wie die anatomische Betrachtung, daß der Druckwirkung einer geschwollenen Thymus zuerst und vor allem die großen Gefäße unterliegen. In welcher Weise diese Druckwirkung klinisch in die Erscheinung tritt, zeigte der Fall deutlich, nämlich in Anfällen schwerster Störung des Blutumlaufs mit Cyanose und schwindendem Puls. Und durch diesen Komplex von Symptomen tritt der Fall ebenso wie durch seinen Ausgang in $\mathrm{Be}-$ ziehung zu den "plötzlichen Todesfällen der Kinder bei Thymushypertrophie“. Es muß einem dabei der Gedanke kommen, ob denn wirklich alle diese Todesfälle so ganz ohne Vorboten eintreten, ob nicht bei manchen dieser Kinder wie bei meinem Kinde leichtere Anfälle dem tödlichen vorausgingen, die aber wegen ihrer Kürze unbemerkt blieben. Der 'Tod durch 'Thymushypertrophie ist jedenfalls in meinem Falle, wie in allen übrigen, eher die Folge des Druckes auf die Gefäße, als des Druckes auf die Trachea. Natürlich braucht man nicht gleich $\mathrm{zu}$ verallgemeinern und $\mathrm{zu}$ sagen, daß alle plötzlichen Todesfälle kleiner Kinder durch Druck der Thymus auf die großen Gefäße verursacht sind. 